\title{
Feasibility Study of Improved Patch Group Prior Based Denoising (PGPD) Technique with Medical Ultrasound Imaging System
}

\author{
Seung Hun Kim, Kanghyen Seo, Seong Hyeon Kang, Jong Hun Kim, Won Ho Choi, and Youngjin Lee* \\ Department of Radiological Science, Eulji University, 553, Sanseong-daero, Sujeong-gu, Seongnam-si, \\ Gyeonggi-do 13135, Republic of Korea
}

(Received 2 February 2017, Received in final form 21 February 2017, Accepted 22 February 2017)

\begin{abstract}
The purpose of this study was to quantitatively evaluate image quality using intensity profile, coefficient of variation (COV), and peak signal to noise ratio (PSNR) with respect to noise reduction techniques in the ultrasound images. For that purpose, we compared with the median filter, Rudin-Osher-Fatemi (ROF), Anscombe and proposed patch group prior based denoising (PGPD) techniques. To evaluate image quality, the Shepp-Logan phantom and the ultrasound image were acquired using simulation and experiment, respectively. According to the results, the difference of intensity profile using PGPD technique is lowest compared with original Shepp-Logan phantom. In simulation, the measured COV was 0.249, 0.198, 0.198, 0.177, and 0.080 using noisy, median, ROF, Anscombe and PGPD technique, respectively. Also, in experimental image, the measured COV was $0.245,0.230,0.231,0.242$ and 0.187 using noisy, median, ROF, Anscombe and PGPD technique, respectively. Especially, when we used PGPD technique, the PSNR has highest value in both simulation and experiment. In this study, we performed simulation and experiment study to compare various denoising techniques in the ultrasound image. We can expect the PGPD technique to improve in medical diagnosis with excellent noise reduction.
\end{abstract}

Keywords : medical diagnostic and ultrasound imaging, noise reduction technique, patch group prior based denoising (PGPD) technique, medical application

\section{Introduction}

Ultrasound refers to the sound wave over $20,000 \mathrm{~Hz}$. Especially, the 1-20 MHz is the range used for body scanning and medical diagnosis [1]. Ultrasound scanner has developed steadily since 1949 , and has three major advantages compared to other medical imaging equipment. Firstly, the ultrasound wave is non-invasive and nondestructive because of physical performance of sound beam. Secondly, the depiction of moving organs such as blood flow, blood vessel, and valve of the heart is precise. Thirdly, it is easier to obtain tomographic image with real time. As above-mentioned major advantages, the ultrasound scanner is widely used in the field of medical imaging with various diagnostic ranges.

However, one of the main problems is the image noise in the ultrasound image. The noise is inevitable in this

(C)The Korean Magnetics Society. All rights reserved.

*Corresponding author: Tel: $+82-31-740-7264$

Fax: +82-31-740-8352, e-mail: radioyoungj@gmail.com image and influenced on the diagnostic accuracy between lesion and mass. In particular, the speckle noise is most often when we performed ultrasound scanning. Therefore, the denoising technique is essential for improvement of image quality in the ultrasound image. Many studies demonstrated that the low pass filter is very useful noise reduction [2-4].

Although this filter can reduce noise with smoothing high frequency area, the edge is partially removed. To improve edge detection, total variation (TV), Rudin-OsherFatemi (ROF) and Anscombe denoising techniques were developed [5]. In recent years, not only excellent noise reduction but also maintaining the quality of image by applying patch based denoising technique is on the main research topic [6,7]. Nonlogal self-similarity (NSS) is one of the best algorithms among patch based denoising techniques. However, image restoration of processed image using NSS technique is still an open problem [8]. To solve this problem, patch group prior based denoising (PGPD) technique has been developed. The PGPD technique is based on NSS that adds Gaussian filter to improve 
noise reduction rate. The process is composed of series of steps where similar pixel is grouped together, and then the average value is deducted. After that, the noise is removed using Gaussian mixture model (GMM). Finally, the average data is added to image using GMM [9].

The purpose of this study was to quantitatively evaluate and compare image qualities with respect to the denoising techniques. For that purpose, we calculated intensity profile, coefficient of variation (COV) and peak signal to noise ratio (PSNR) using both simulation and experiment.

\section{Materials and Methods}

\subsection{Proposed PGPD technique}

A PG based GMM (PG-GMM) denoising technique was suggested by C. M. Bishop in 2006 [10]. This technique is consisted of expectation maximization (EM) algorithm, which is iterative processing method using maximum likelihood estimation in statistical model, with Gaussian filter [11]. Based on EM algorithm, PG-GMM with PG using combination between local patch and similar patch is calculated as follows:

$$
P G(k \mid \bar{Y})=\frac{\prod_{m}^{M} N\left(\bar{Y}_{m} \mid 0, \Sigma_{k}+\sigma^{2} W\right)}{\Sigma_{l=1}^{K} \Pi_{m}^{M} N\left(\bar{Y}_{m} \mid 0, \Sigma_{l}+\sigma^{2} W\right)}
$$

where $P G(k \mid \bar{Y})$ is the component with the highest probability, $N$ is the number of $P G, M$ is patch number in $P G, \bar{Y}$ is subtraction image and $\sigma$ is the standard deviation. The kth covariance matrix will become $\Sigma_{k}+\sigma^{2} W$ ( $W$ is the identity matrix) when $\sigma^{2}$ is the variance of image. Also, we can find PG form similar to the each local patch by using a noisy image and $\bar{Y}$ in $P G(k \mid \bar{Y})$ equation. When we applied $P G(k \mid \bar{Y})$ equation based on log-likelihood, this equation may be converted as follows:

$$
\ln P G(k \mid \bar{Y})=\Sigma_{m}^{M} \ln N\left(\bar{Y}_{m} \mid 0, \sum_{k}+\sigma^{2}\right)-\ln C
$$

where $C$ is the denominator in above equation. Then, denoising by weighted sparse coding is required when it finish selecting component. Practically, $P G$ denotes the noise with Gaussian distribution of the similar patches in each group. Thus, we can remove noise by using singular value decomposition.

$$
Z=D A D^{T}
$$

where $D$ is an orthonormal matrix consists of the eigenvectors of $Z, A$ is the diagonal matrix of eigenvalues and $D^{T}$ is unitary matrix. We used $\overline{y_{m}}$ as the dictionary using sparse encoding $\left(\overline{y_{m}}=D \alpha+c\right.$, where $\alpha$ is the coefficients for sparse coding vector and $c$ is the corrupted noise).
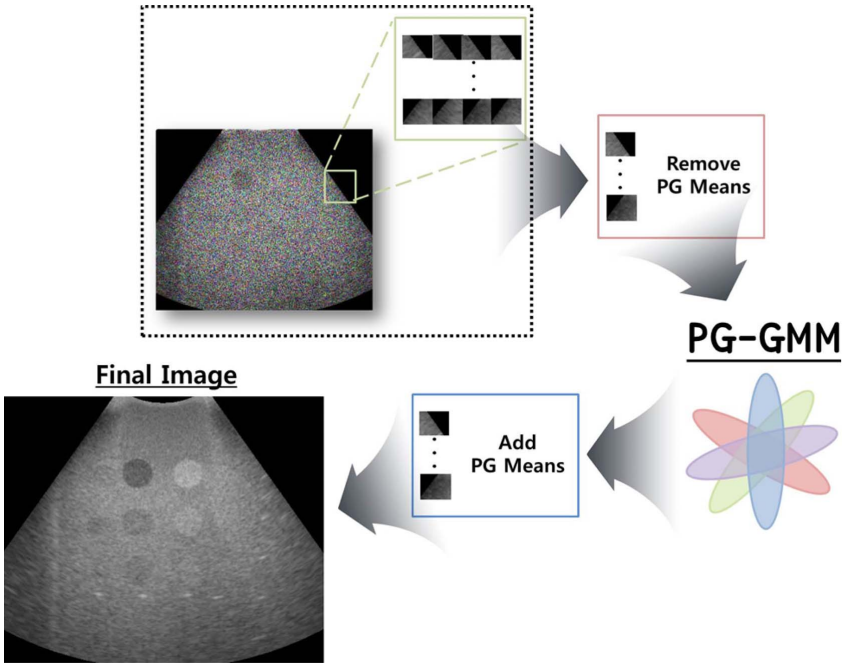

Fig. 1. (Color online) A PG is formed by grouping the similar patches to a local patch in a large enough neighborhood. Next PG Means are removed in PG. A PG based GMM (PG-GMM) denoising algorithm is developed to learn the NSS prior for the PGs. PG-GMM will provide dictionaries as well as regularization parameters, and a simple weighted sparse coding model is developed for image denoising. Finally PG is finished by PG-GMM that add PG-Means.

Also, we proposed a weighting vector (w) for $\alpha$.

$$
\min _{\alpha}\left\|\bar{Y}_{m}-D \alpha\right\|_{2}^{2}+\left\|w^{T} \alpha\right\|_{1}
$$

When we optimized solution, we estimated viewpoint of Maximum A-Posterior (MAP).

$$
\hat{\alpha}=\arg \max _{\alpha}\left\{\ln P\left(\alpha \mid \bar{y}_{m}\right)\right\}
$$

Also, we represented equation by Bayes' formula [12].

$$
\hat{\alpha}=\arg \max _{\alpha}\left\{\ln P\left(\bar{y}_{m} \mid \alpha\right)+\ln P(\alpha)\right\}
$$

( $\ln P\left(\bar{Y}_{m} \mid \alpha\right)$ using log-likelihood term is characterized by the noise statistics)

This log-likelihood term is estimated to be Gaussian with $\sigma$ and is calculated as follows:

$$
P\left(\bar{Y}_{m} \mid \alpha\right)=\frac{1}{\sqrt{2 \pi \sigma^{2}}} \exp \left(-\frac{1}{2 \sigma^{2}}\left\|\bar{y}_{m}-D \alpha\right\|_{2}^{2}\right)
$$

Based on Laplacian distribution based on probability theory, we assumed $P(\alpha)$ [13].

$$
P(\alpha)=\prod_{i=1}^{P^{2}} \frac{c}{\sqrt{2} \lambda_{i}} \exp \left(-\frac{c \sqrt{2} \mid \alpha_{i}}{\lambda_{i}}\right)
$$

where $\alpha_{i}$ is the coding coefficient for $\bar{Y}_{m}$ over the ith eigenvector in $D, \lambda_{i}$ is $A_{i}^{1 / 2}$ and $c$ is a constant. Then, putting $P\left(\bar{Y}_{m} \mid \alpha\right)$ and $P(\alpha)$ into $\hat{\alpha}$, we changed formula from equation $\hat{\alpha}$ and we can unite each patch. 


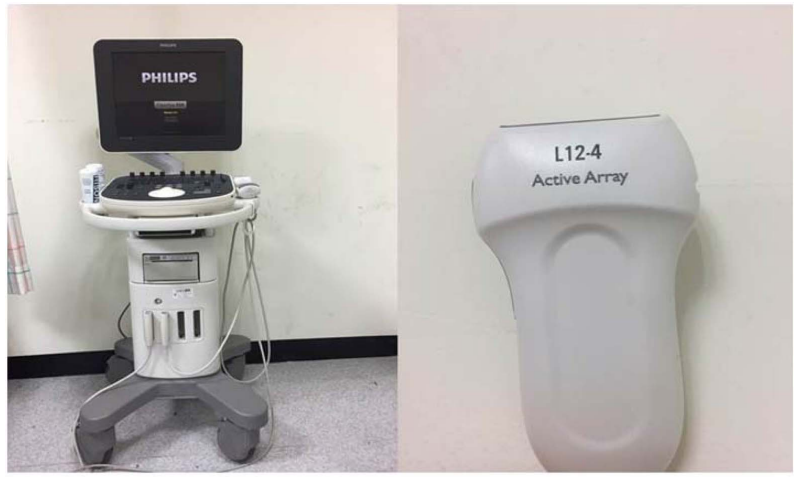

(a)

(b)

Fig. 2. (Color online) The photo of used (a) ultrasound device and (b) linear probe.

\subsection{Simulation condition and used ultrasound devices} for experimental study

We implemented propose algorithm with Shepp-Logan phantom using the MATLAB (Version 8.3) program for the simulation study [14]. Then, we performed experimental study using ultrasound device (ClearVue 550, Philips) and linear probe in this study (Fig. 2). The used probe is linear probe with $7.5 \mathrm{MHz}$ frequency and we acquired images for thyroid.

\subsection{Evaluation of image quality}

We evaluated three performance evaluation methods to compare denoising techniques: (1) intensity profile, (2) COV, and (3) PSNR. To evaluated similarity between original and denoising images, we acquired intensity profile in the simulation at same area. Also, we evaluated COV that calculated standard variation of population divided by mean of population and PSNR in both simulation and experiment. The COV and PSNR calculated as follows:

$$
\begin{aligned}
& C O V=\frac{\sigma}{\mu} \\
& P S N R=10 \cdot \log _{10} \frac{\max (o(x, y))^{2}}{\frac{1}{n_{x} \times n_{y}} \times \Sigma_{0}^{n_{x}-1} \Sigma_{0}^{n_{y}-1}[o(x, y)-t(x, y)]^{2}}
\end{aligned}
$$

where $\sigma$ and $\mu$ are standard variation and mean, respectively, $o(x, y)$ is original image, $\max (o(x, y))$ is maximum $\mathrm{dB}$ in the image, $n_{x} \times n_{y}$ is size of image, and $t(x, y)$ is denoising images.

\section{Results and Discussion}

We evaluated intensity profile, COV and PSNR using different denoising techniques. Figure 3 and 4 show the

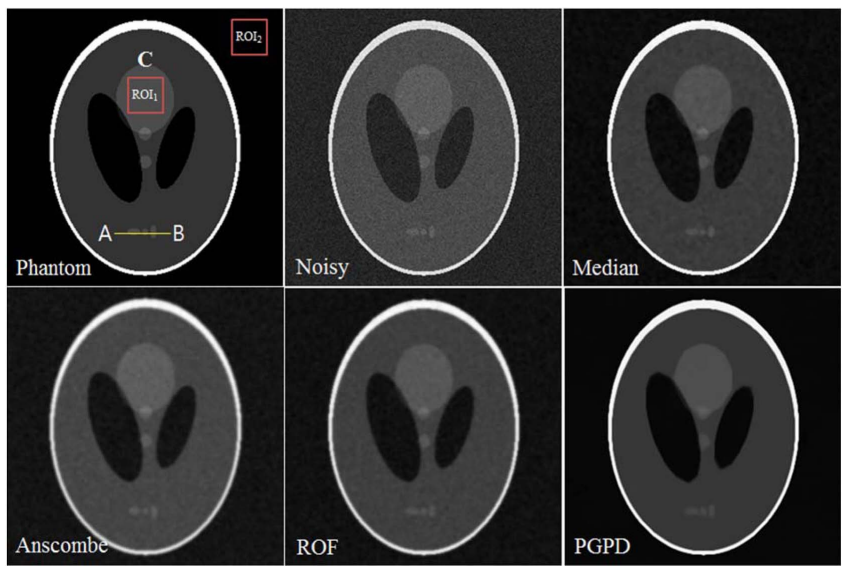

Fig. 3. (Color online) The simulation images for the applied various denoising techniques.

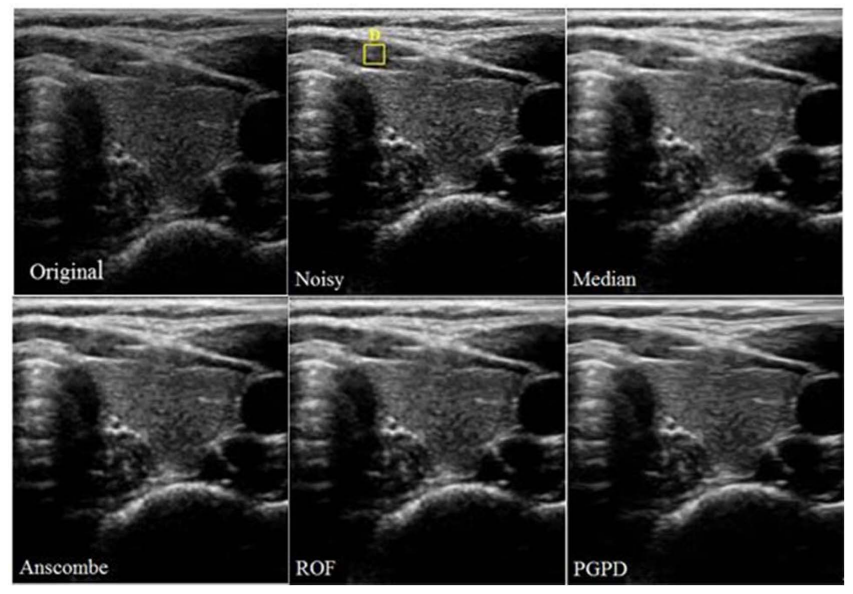

Fig. 4. (Color online) The experimental ultrasound images for the applied various denoising techniques.

acquired Shepp-Logan phantom images and real ultrasound images for thyroid using linear probe, respectively. We acquired intensity profile based on A-B line (Fig. 3) in original and all of the denoising images. Comparing the part to the intensity profile with different denoising techniques is the Fig. 5. As a result, PGPD technique is best result for excellent similarity between original phantom and denoising images.

Figure 6 shows the COV results with respect to the denoising techniques. We acquired COV based on ROI 1 (Fig. 3) in noisy and all of the denoising images. In simulation, the measured COV was 0.249 , 0.198, 0.198, 0.177 and 0.08 using noisy, median, ROF, Anscombe and PGPD, respectively. According to the simulation results, the PGPD technique had approximately $1 / 3$ less noise compared to the noisy image, and had approximately $1 / 2$ less data value than other denoising techniques. Also, in experiment, the measured COV was $0.245,0.230,0.231$, 


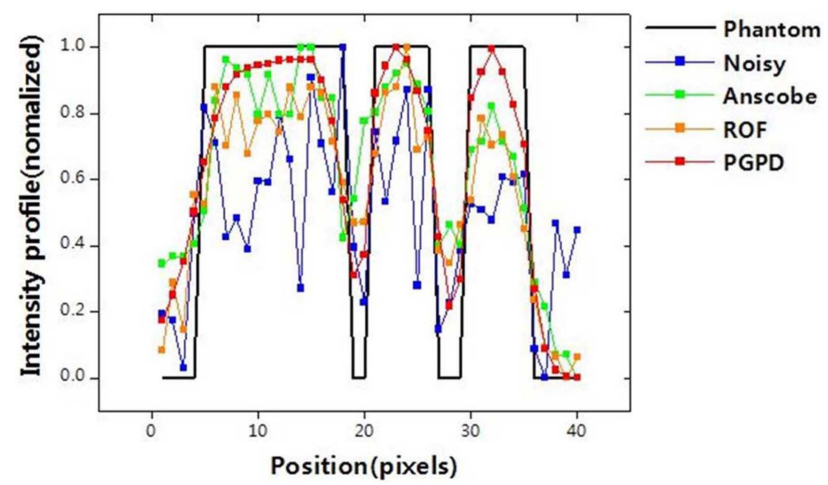

Fig. 5. (Color online) The results for the intensity profile using different denoising techniques.

0.242 and 0.187 using noisy, median, ROF, Anscombe and PGPD, respectively. According to the experimental result, the PGPD technique is 0.8 and 0.85 times improvement of COV than noisy image and other denoising techniques, respectively. The normalized COV results are shown in Fig. 7.

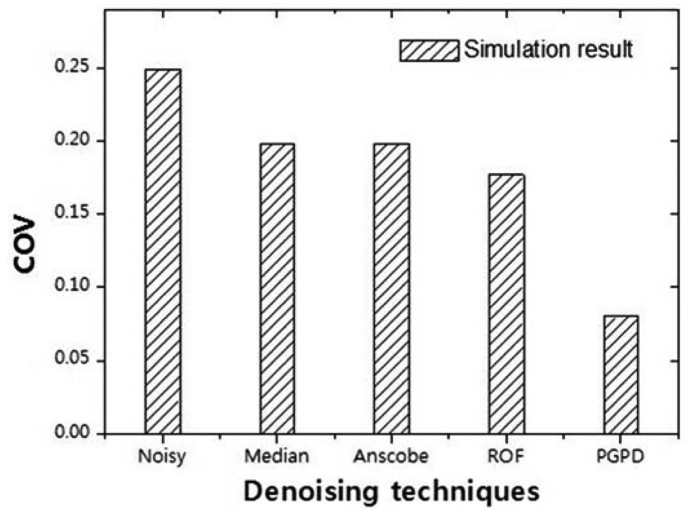

(a)
Figure 8 shows the PSNR results with respect to the denoising techniques. In simulation, the measured PSNR was $10.80,10.88,8.94,11.15$ and 75.93 using noisy, median, ROF, Anscombe and PGPD, respectively. According to the simulation results, the PGPD technique is approximately 7.5 times higher than that of the noisy image. Also, this technique is 7 to 9 times higher than that of the other technique except for noisy. In experiment, the measured PSNR was 13.66, 13.70, 11.51, 13.83 and 82.40 using noisy, median, ROF, Anscombe, and PGPD, respectively. According to the experimental result, the PGPD technique is 6 and 6-7 times improvement of PSNR than noisy image and other denoising techniques, respectively.

In summary, PGPD technique has better image quality in intensity profile, COV, and PNSR results compared to other algorithms. This is because PGPD technique has different characteristics, which patch group finds similar pixels to replace original image group, from other algorithms. Furthermore, we can acquire improved results for denoising efficiency because PGPD technique applies Gaussian filter on the patch group.

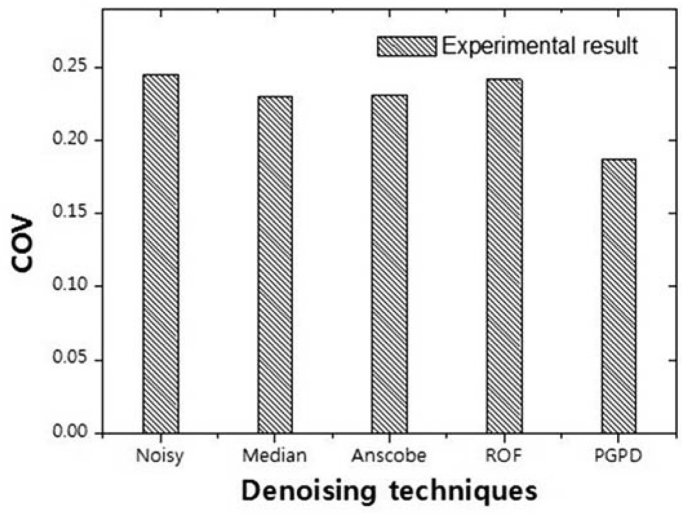

(b)

Fig. 6. The COV results for (a) simulation and (b) experiment as function of denoising techniques.

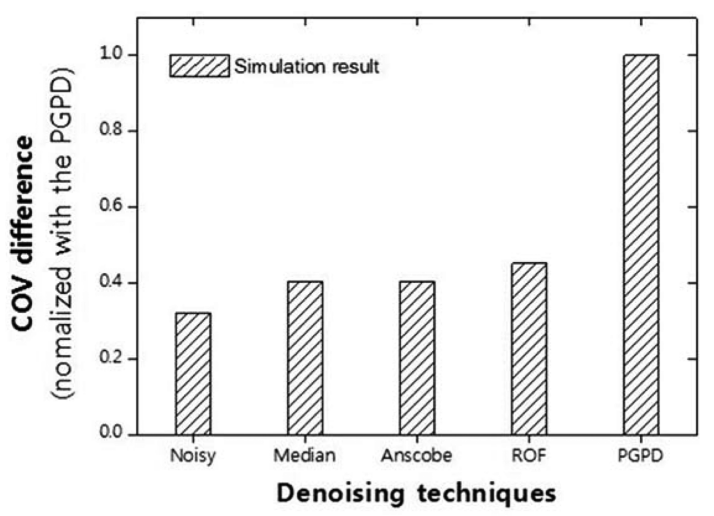

(a)

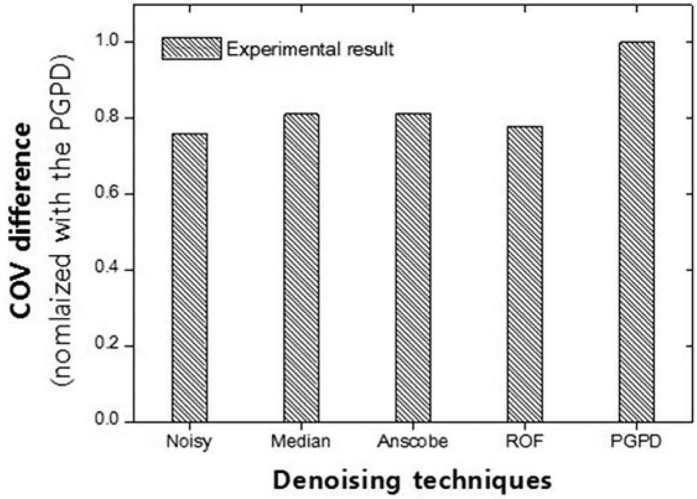

(b)

Fig. 7. The normalized COV results for (a) simulation and (b) experiment. 


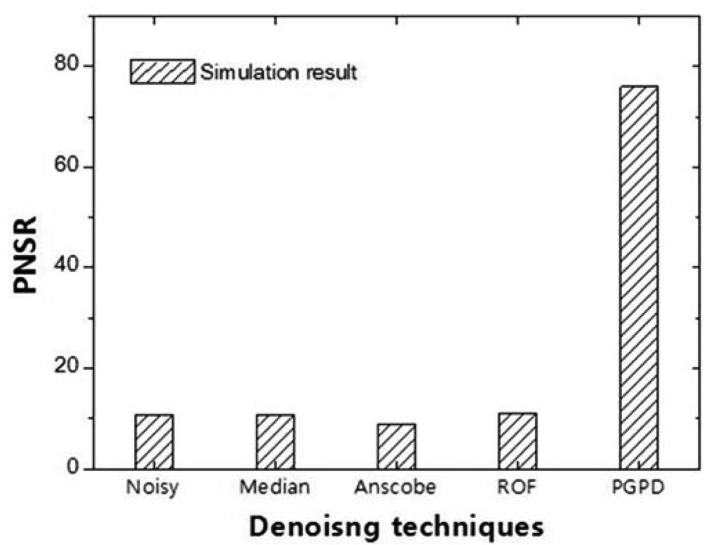

(a)

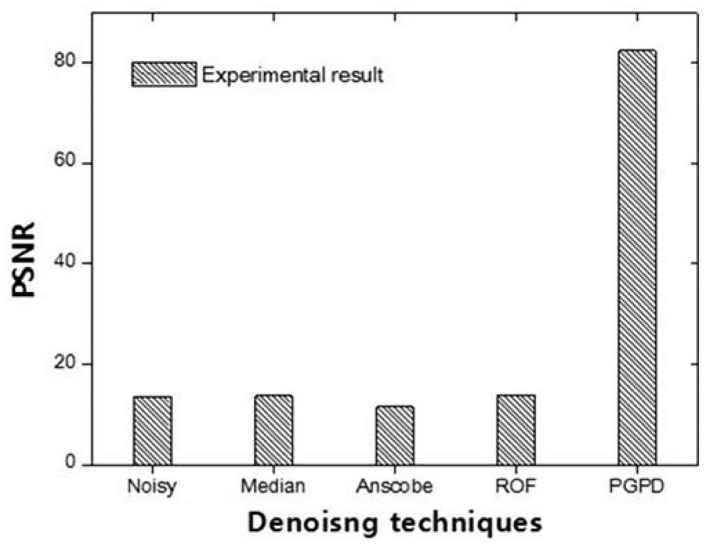

(b)

Fig. 8. The PSNR results for (a) simulation and (b) experiment as function of denoising techniques.

Especially, COV was used quantitatively evaluation of noise because it was proportionate to noise. Also, PSNR is most commonly used to measure the image quality, while it cannot reflect some visual features such as the preservation of edges and other geometric features. Thus, COV and PSNR with PGPD technique were stabilized because of efficient noise reduction.

\section{Conclusion}

The various denoising techniques are frequently used for accurate diagnosis in field of pre-clinical or clinical imaging system. This research proves the excellence of PGPD technique based on patch group in comparison of other frequently used denoising techniques in ultrasound imaging system. By grouping similar patches, we can efficiently removing the minor and major noise. Thus, since our proposed technique provides high quality by superb noise reduction, the clearer and precise image scanning would be possible.

\section{Acknowledgment}

This paper was supported by Eulji University in 2017 and was supported by the National Research Foundation of Korea (NRF-2016R1D1A1B03930357).

\section{References}

[1] G. J. Czarnotta and M. C. Kolios, Imaging in Medicine 2, 17 (1020).

[2] P. Hellier, C. Kervrann, and C. Barillot, IEEE Transactions Image Processing 18, 2221 (2009).

[3] K. Djemal, IEEE International Conference on Image Processing 3, 357 (2005).

[4] L. I. Rudin, S. Osher, and E. Fatemi, Physica D 60, 259 (1992).

[5] S. Suresh, G. R. Suresh, and R. Sukanesh, International Journal of Computer Theory and Engineering 1, 7 (2009).

[6] A. Buades, B. Coll, and J. M. Morel, Multiscale Modeling \& Simulation 4, 490 (2005).

[7] P. Chatterjee and P. Milanfar, IEEE Transactions on Image Processing 19, 895 (2010).

[8] A. Buades, B. Coll, and J. M. Morel, IEEE Computer Society Conference on Computer Vision and Pattern Recognition 2, 60 (2005).

[9] J. Xu, L. Zhang, W. Zuo, D. Zhang, and X. Feng, The IEEE International Conference on Computer Vision 244 (2015).

[10] C. M. Bishop, Springer, New York (2006).

[11] CF Jeff Wu, The Annals of Statistics 11, 95 (1983).

[12] D. Isa, Lam H. Lee, V. P. Kallimani, and R. RajKumar, IEEE Transactions on Knowledge and Data Engineering 20, 1264 (2008).

[13] P. Burt and E. Adelson, IEEE Transactions on Communications 31, 532 (1983).

[14] L. A. Shepp and B. F. Logan, IEEE Transactions on Nuclear Science 21, 21 (1974). 